\title{
CHIRAL MESON MASSES AT FINITE TEMPERATURE AND DENSITY
}

\author{
A. Barducci, R. Casalbuoni, G. Pettini \\ Dipartimento di Fisica, Università di Firenze, I-50125 Firenze, Italy \\ and Istituto Nazionale di Fisica Nucleare, Sezione di Firenze, I-50125 Firenze, Italy \\ R. Gatto \\ Département de Physique Théorique, Université de Genève, CH-1211 Genève 4, Switzerland \\ UGVA-DPT 2000/08-1087 \\ Firenze Preprint - DFF - 359/06/2000
}

\begin{abstract}
The ratio of the sigma mass to the pion mass at finite temperatures and densities provides for a quantitative signal of chiral symmetry breaking. We calculate this ratio by using an extension to finite chemical potential of the field theoretic composite operator formalism as applied to QCD. The calculation is limited to regions of the phase diagram where only quark-antiquark condensates dominate (no quark-quark condensates) and it confirms the expected behaviours. In particular the sigma becomes an essentially stable particle in a narrow region bordering the transition line from broken to restored chirality. This pattern is qualitatively the same both for the region where the transition is of second order as for the region where it is of first order (apart from the discontinuities expected in the latter case).
\end{abstract}

PACS number(s): 12.38.Aw, 05.70.Jk, 11.30.Rd, 12.38.Mh, 11.10.Wx

Typeset using REVTEX 


\section{INTRODUCTION}

Since the original discussion by Nambu [1] in 1960, chiral symmetry breaking has played a crucial role in the theory of strong interactions. In 1974 Lee and Wick [2] proposed that at high densities chiral symmetry may be restored. Restoration at high temperatures was discussed in the same period by Dolan and Jackiw [3] and by Weinberg [4]. Condensation of quark-antiquark pairs plays the main role in chiral symmetry breaking, except for very high densities where quark-quark condensates are expected to play the important role. In this note we do not consider this very high density region and limit ourselves to densities such that only quark-antiquark condensates are important. Such a region is the one important in hadronic physics, and chiral restoration from this region is expected to be reachable with high energy heavy ion collisions [5]. Chiral transition through the quark-antiquark order parameter is also expected to be of great relevance for the physics of the early universe [6].

A great number of studies has been carried out on the expected QCD phase diagram (see for instance [7]). For a review of the subject see the recent paper by Rajagopal [8]

Lattice calculations are rapidly progressing and they will with time provide for a complete approach (a review can be found for instance in Ref. [9]). Many theoretical approaches use the Nambu-Jona-Lasinio model and different dynamical approximations for quantitative indications on the chiral transition (see for instance [10]).

Besides the chiral transition also the deconfinement transition takes place. A single transition is generally expected to occur, rather than separate transitions for deconfinement and chiral-symmetry (see for instance for a heuristic argument Ref [1]). Theoretically one has to deal with different order parameters. Moreover they cover extreme ranges in opposite domains. The transition from the confined to the non-confined phase is often described in terms of the thermally averaged Polyakov loop which applies particularly in the limit of infinite quark masses. For the chiral symmetry transition the typical order parameters are the thermally averaged quark-antiquark bilinears (at least up to not very high densities) which apply mostly in the limit of vanishing quark masses. 
The current quark mass violates explicitly the chiral symmetry whose restoration characterizes the phase transition. Its role is formally analogous to that of the external magnetic field in the ferromagnetic transition. As long as the mass is small, such as for $u$ and $d$ quarks, the notion of phase transition can be approximately useful.

Our study uses a composite operator formalism for both finite temperature and finite density. It is based on constructing the effective action for the composite operators 12,13] in the presence of temperature and chemical potential. We focus on the study of the ratio of the scalar to pseudoscalar mass $M_{\sigma} / M_{\pi}$ in the plane of temperature and chemical potential. The ratio might have a physical interest as a possible experimental signature for the transition, although it is too early for assessing its usefulness in these regards. It has anyway a theoretical interest as it constitutes a significant parameter for the degree of chiral symmetry breaking at finite temperatures and densities. The ratio is equal to unity when chiral symmetry is restored (we neglect for the moment the small $u$ and $d$ current quark masses). In the hadronic phase, at zero temperature and small density, in the broken chirality phase, the pion is a goldstone of vanishing mass, while the sigma has a finite mass from the chiral condensation. The ratio of the scalar to the pseudoscalar mass is thus expected to decrease from very large values to a value of one at the chiral transition (the $u$ and $d$ current quark masses only slightly change this picture).

A physical consequence of this behaviour is that, before approaching the critical values for the transition, by increasing together or separately temperature and density, for the sigma particle there is not any phase space left for its decay into two pions. Its main source of instability is thus suppressed when approaching the phase border in the two dimensional plane of temperature and chemical potential. Consequently the sigma becomes an almost stable particle with dominant decay channel [14] that into two photons. The suggested experimental signature would thus be in that region a bump in the invariant mass of two photons. We do not know at this stage how this will be visible in heavy ion collisions.

On the other hand it is worthwhile to recall that indications on the sigma at zero temperature and density have been rather elusive just because of the very large width the sigma 
has in that case. It has not been demonstrated so far that the pion-pion phase shift passes through 90 degrees in a region below some 700-800 MeV, suggesting that the large width may mask the overall effect. Also it is well-known that strong rescattering effects in different channels make the interpretation of the low energy phenomenology in the $0^{++}$channel not easy [15]. We just mention that a recent experiment by the E91 collaboration brings evidence for a light and broad scalar resonance in the D-decay channel into three pions [16] and that a simple model makes plausible the interpretation in terms of a sigma resonance [17]. For further phenomenological discussions on the sigma resonance in hadronic physics we refer to the papers posted in relation to the recent conference on "Possible existence of a light sigma resonance and its implications to hadron physics", held in Kyoto, June 11-14 (for a review see the summary talk by Tornqvist [18]). We also mention that there is been recently discussion as to the possibility of a glueball interpretation of the sigma resonance and for this we refer to a recent talk by Pennington disfavoring such an interpretation [19].

We recall that the QCD phase diagram, even for massless quarks, is known to have a richer structure when studied in the plane of $\mathrm{T}$ and chemical potential, as one expects both types of transitions, of second order for low chemical potentials and of first order beyond some critical value of the chemical potential. Besides, for large chemical potential a color superconductivity phase should occur, but here we limit ourselves to consequences of quarkantiquark condensations and assume not yet to be in a region where quark-quark condensates appear. The separation along the critical line between the two types of transitions defines a tricritical point which has been the subject of extensive studies [20,21]. As far as the particular subproduct of the present calculation, that is finding the region in the phase diagram where the sigma becomes of such a low mass to be no longer able to decay into two pions and thus appearing as a narrow state, we find that, independently on the type of transition, this region is anyway confined to a small strip near the transition line. What changes of course is that when passing through the transition line one finds for the the ratio of the sigma to the pion mass a discontinuity in the case when the transition is of first order.

In Section II we recall the main steps to evaluate the effective potential in the composite 
operator formalism. Then we derive the expressions for the pion and $\sigma$-meson masses at finite temperature and density in our model. Finally we obtain a formula for their ratio and we show the numerical results for typical behaviours when crossing the lines of second and first order phase transitions.

\section{EFFECTIVE ACTION}

The zero temperature Euclidean effective action for an $S U(N)$ QCD-like gauge theory is (we follow [12])

$$
\Gamma(\boldsymbol{\Sigma})=-\operatorname{Tr} \ln \left[\mathbf{S}_{0}^{-1}+\frac{\delta \Gamma_{2}}{\delta \mathbf{S}}\right]-\operatorname{Tr}[\boldsymbol{\Sigma} \mathbf{S}]-\Gamma_{2}(\mathbf{S})+\text { counterterms }
$$

where

$$
\mathbf{S}_{0}^{-1}=(i \hat{p}-\mathbf{m})
$$

$\mathbf{m}$ is the bare quark mass matrix and $\Gamma_{2}(\mathbf{S})$ is the sum of all the two-particle irreducible vacuum diagrams with fermionic propagator $\mathbf{S}$ and $\boldsymbol{\Sigma}=-\delta \Gamma_{2} / \delta \mathbf{S}$. One has at two-loop level

$$
\Gamma_{2}=\frac{1}{2} \operatorname{Tr}(\mathbf{S} \Delta \mathbf{S})
$$

where $\Delta$ is the gauge boson propagator, so that

$$
\Sigma=-\Delta \mathbf{S}
$$

One can therefore rewrite Eq. (11)

$$
\Gamma(\boldsymbol{\Sigma})=-\operatorname{Tr} \ln \left[\mathbf{S}_{0}^{-1}-\boldsymbol{\Sigma}\right]+\frac{1}{2} \operatorname{Tr}\left(\boldsymbol{\Sigma} \Delta^{-1} \boldsymbol{\Sigma}\right)+\text { counterterms }
$$

A parametrization for $\boldsymbol{\Sigma}$ employed in [12] was

$$
\mathbf{\Sigma}=\left(\mathbf{s}+i \gamma_{5} \mathbf{p}\right) f(k) \equiv \Sigma_{s}+i \gamma_{5} \boldsymbol{\Sigma}_{p}
$$

with $\mathbf{s}$ and $\mathbf{p}$ scalar and pseudoscalar constant fields respectively and with a suitable Ansatz for $f(k)$. 
From Eq. (5) (see Ref. [12]) one obtains the effective potential

$$
\begin{aligned}
V=\frac{\Gamma}{\Omega}= & -\frac{8 \pi^{2} N}{3 C_{2} g^{2}} \int \frac{d^{4} k}{(2 \pi)^{4}} \operatorname{tr}\left[\boldsymbol{\Sigma}_{s} \square_{k} \boldsymbol{\Sigma}_{s}+\boldsymbol{\Sigma}_{p} \square_{k} \boldsymbol{\Sigma}_{p}\right]- \\
& -N \operatorname{Tr} \ln \left[i \hat{k}-\left(\mathbf{m}+\boldsymbol{\Sigma}_{s}\right)-i \gamma_{5} \boldsymbol{\Sigma}_{p}\right]+\delta Z \operatorname{tr}(\mathbf{m} \mathbf{s})
\end{aligned}
$$

where $C_{2}$ is the quadratic Casimir of the fermion representation (for $S U(3)_{c} C_{2}=4 / 3$ ), $g$ is the gauge coupling constant and $\Omega$ the four-dimensional volume. Furthermore

$$
\begin{aligned}
\boldsymbol{\Sigma}_{s} & \equiv \frac{\lambda_{\alpha}}{\sqrt{2}} s_{\alpha} f(k) \\
\boldsymbol{\Sigma}_{p} & \equiv \frac{\lambda_{\alpha}}{\sqrt{2}} p_{\alpha} f(k) \\
\mathbf{m} & \equiv \frac{\lambda_{\alpha}}{\sqrt{2}} m_{\alpha}
\end{aligned}
$$

with $\alpha=0, \cdots, 8 ; \quad \lambda_{0}=\sqrt{2 / 3} I$ and $\lambda_{i}(i=1, \cdots, 8)$ are the Gell-Mann matrices.

For $f(k)$ we have chosen as a variational Ansatz [20]

$$
f(k)=\frac{M^{3}}{k^{2}+M^{2}}
$$

where $M$ is a momentum scale expected to be of the order of $\Lambda_{Q C D}$.

The extension to non zero temperature $T$ and non zero chemical potential $\mu$ corrects the effective potential for a quark of mass $m$ to the form

$$
\begin{aligned}
V=\frac{\Gamma}{\Omega}= & -\frac{8 \pi^{2} N}{3 C_{2} g^{2}(T, \mu)} \int \frac{d^{4} k}{(2 \pi)^{4}}\left[\Sigma_{s} \square_{k} \Sigma_{s}+\Sigma_{p} \square_{k} \Sigma_{p}\right]- \\
& -2 N \sum_{n=-\infty}^{+\infty}(-)^{n} \int \frac{d^{4} k}{(2 \pi)^{4}} \ln \left[\bar{k}^{2}+\left(m+\Sigma_{s}\right)^{2}+\Sigma_{p}^{2}\right] e^{i n \frac{k_{0}}{T}}+\delta Z m s
\end{aligned}
$$

where $\bar{k}^{\nu} \equiv\left(k_{0}+i \mu, \mathbf{k}\right)$ and we have used the Poisson formula, equivalent to the application of standard imaginary-times rules [3], 20,22].

In Eq. (10)

$$
\Sigma_{s}=s f(\bar{k})
$$

and analogously for $\Sigma_{p}$. The coupling constant depends on $T$ and $\mu$ as 


$$
\frac{2 \pi^{2}}{g^{2}(T, \mu)} \equiv c(T, \mu) \equiv c_{0}+c_{1}(T, \mu)=c_{0}+\frac{\pi^{2}}{b} \ln \left(1+\xi \frac{T^{2}}{M^{2}}+\zeta \frac{\mu^{2}}{M^{2}}\right)
$$

with $b=24 \pi^{2} /\left(11 N-2 n_{f}\right)$, and we will discuss the determination of the parameters $c_{0}, M, \xi$ and $\zeta$ later on. Also for non vanishing $T$ and $\mu$ we impose the normalization

$$
\left.\lim _{m \rightarrow 0} \frac{\partial V}{\partial\left(m\langle\bar{\psi} \psi\rangle_{0}\right)}\right|_{\min }=1
$$

The following relation relates the minimum of the potential $\bar{s}(T, \mu)$ to the condensates $\langle\bar{\psi} \psi\rangle_{T, \mu}$

$$
\langle\bar{\psi} \psi\rangle_{T, \mu}=\frac{N M^{3}}{g^{2}(T, \mu)} \bar{s}(T, \mu)
$$

When using physical pion fields with their proper normalization, the normalization condition of Eq. (13) is equivalent to the Adler-Dashen formula

$$
M_{\pi}^{2}(T, \mu) f_{\pi}^{2}(T, \mu)=-2 m\langle\bar{\psi} \psi\rangle_{T, \mu}
$$

\section{MASSES}

To derive the masses we use canonical fields

$$
\begin{aligned}
\varphi_{\sigma} & =a s \\
\varphi_{\pi} & =a p \\
a & =-\frac{f_{\pi}}{\sqrt{2} \bar{s}}
\end{aligned}
$$

and we obtain

$$
\begin{aligned}
& M_{\sigma}^{2}=\left.\frac{\partial^{2} V}{\partial \varphi_{\sigma}^{2}}\right|_{\min }=\left.\frac{1}{a^{2}} \frac{\partial^{2} V}{\partial s^{2}}\right|_{\min }=\left.2 \frac{\bar{s}^{2}}{f_{\pi}^{2}} \frac{\partial^{2} V}{\partial s^{2}}\right|_{\min } \\
& M_{\pi}^{2}=\left.\frac{\partial^{2} V}{\partial \varphi_{\pi}^{2}}\right|_{\min }=\left.\frac{1}{a^{2}} \frac{\partial^{2} V}{\partial p^{2}}\right|_{\min }=\left.2 \frac{\bar{s}^{2}}{f_{\pi}^{2}} \frac{\partial^{2} V}{\partial p^{2}}\right|_{\min }
\end{aligned}
$$

where $\bar{s}$ is the minimum of the effective potential in presence of the quark bare mass $m$. 
In the following we use the minimum condition

$$
\begin{aligned}
& \frac{\partial V}{\partial s}=0 \rightarrow \\
& \frac{N}{\bar{s}}\left[-2 c(T, \mu) \int \frac{d^{4} k}{(2 \pi)^{4}} \bar{\Sigma}_{s} \square_{k} \bar{\Sigma}_{s}\right. \\
& \left.-4 \sum_{n=-\infty}^{+\infty}(-)^{n} \int \frac{d^{4} k}{(2 \pi)^{4}} \frac{\left(m+\bar{\Sigma}_{s}\right) \bar{\Sigma}_{s}}{\left[\bar{k}^{2}+\left(m+\bar{\Sigma}_{s}\right)^{2}+\bar{\Sigma}_{p}^{2}\right]} e^{i n \frac{k_{0}}{T}}+m \delta Z\right]=0 \\
& \frac{\partial V}{\partial p}=0 \rightarrow \quad \bar{p}=0
\end{aligned}
$$

where $\bar{\Sigma}_{s} \equiv \bar{s} f(\bar{k})$. Furthermore we have the normalization condition for the effective potential

$$
\delta Z=N\left[\frac{M^{3}}{2 \pi^{2}} c(T, \mu)+\frac{4}{s_{0}} \sum_{n=-\infty}^{+\infty}(-)^{n} \int \frac{d^{4} k}{(2 \pi)^{4}} \frac{\Sigma_{0}}{\bar{k}^{2}+\Sigma_{0}^{2}} e^{\left.i n \frac{k_{0}}{T}\right]}\right.
$$

with $\Sigma_{0} \equiv s_{0} f(\bar{k})$ and $s_{0}$ is the minimum for the case of a massless quark. The two preceding equations allow for the following expressions for the masses

$$
\begin{aligned}
& M_{\pi}^{2}(T, \mu)=-2 \frac{m}{f_{\pi}^{2}(T, \mu)}\langle\bar{\psi} \psi\rangle_{T, \mu}+ \\
&+8 N \frac{m}{f_{\pi}^{2}(T, \mu)} \sum_{n=-\infty}^{+\infty}(-)^{n} \int \frac{d^{4} k}{(2 \pi)^{4}}\left[\frac{\bar{\Sigma}_{s}}{\bar{k}^{2}+\left(m+\bar{\Sigma}_{s}\right)^{2}}-\right. \\
&-\frac{\bar{s}(T, \mu)}{s_{0}(T, \mu)} \frac{\Sigma_{0}}{\bar{k}^{2}+\Sigma_{0}^{2}} e^{i n \frac{k_{0}}{T}} \\
& M_{\sigma}^{2}(T, \mu)=M_{\pi}^{2}(T, \mu)+\frac{16 N}{f_{\pi}^{2}(T, \mu)} \sum_{n=-\infty}^{+\infty}(-)^{n} \int \frac{d^{4} k}{(2 \pi)^{4}} \frac{\left(m+\bar{\Sigma}_{s}\right)^{2}}{\left[\bar{k}^{2}+\left(m+\bar{\Sigma}_{s}\right)^{2}\right]^{2}} \bar{\Sigma}_{s}^{2} e^{i n \frac{k_{0}}{T}}
\end{aligned}
$$

The parameters $c_{0}$ and $s_{0}(T=\mu=0)$ are fixed by following the procedure of Ref. [12, 13, 20, 23, where one was able to fix the value $c_{0}=0.554$ from the renormalization condition and the gap equation for the effective potential in the limit of small current quark masses. By inserting this value for $c_{0}$ in the gap equation for massless quarks, one finds that chiral symmetry is spontaneously broken (at $T=\mu=0$ ). Actually the origin of $V$ is a local maximum whereas the absolute minimum is at $s_{0}(T=\mu=0)=-4.06$. To determine 
the mass scale $M$ and the quark masses from the experimental data, one has to derive the explicit expressions for the masses and for the decay constants of the pseudoscalar octet mesons which are the pseudo-Goldstone bosons of chiral symmetry breaking. These expressions constitute a system of coupled equations which we have solved by an approximation method. The experimental inputs are the decay constant and mass of the charged pion, the charged kaon mass and the electromagnetic mass difference between the neutral and the charged kaon. The outputs of the numerical fit for the octet meson masses (agreement within $3 \%$ ) are the masses of the $u, d$ and $s$ quarks and the mass parameter $M$. The values we get are the following [12,13,23

$$
\begin{aligned}
M & =280 \mathrm{MeV} \\
m_{u} & =8 \mathrm{MeV} \\
m_{d} & =11 \mathrm{MeV} \\
m_{s} & =181 \mathrm{MeV} \\
m & \equiv \frac{m_{u}+m_{d}}{2}=9.5 \mathrm{MeV}
\end{aligned}
$$

Finally, the parameter $\xi$ in the expression (12) for the running coupling constant has been set to $\xi \simeq 1$ [24] by comparing our model in the low- $T$ and $\mu=0$ regime with the results of Ref. [25], whereas $\zeta$ is left as undetermined [20,26].

With these values for the relevant parameters it is easy to determine the masses of the pseudoscalar and scalar mesons at $T=\mu=0$ (we are using the value $f_{\pi}=93 \mathrm{MeV}$ for the pion decay constant)

$$
\begin{aligned}
& M_{\pi}=135 \mathrm{MeV} \\
& M_{\sigma}=630 \mathrm{MeV}
\end{aligned}
$$

and (again at $T=\mu=0$ ) the fermionic condensates at $M$ for massless and massive quarks

$$
\begin{aligned}
& \langle\bar{\psi} \psi\rangle \simeq(-197 \mathrm{MeV})^{3} \\
& \langle\bar{\psi} \psi\rangle \simeq(-200 \mathrm{MeV})^{3}
\end{aligned}
$$


respectively.

\section{THE RATIO $M_{\sigma}^{2} / M_{\pi}^{2}$}

From Eq. (22) we can calculate the ratio $M_{\sigma}^{2} / M_{\pi}^{2}$. We then use the expression for $f_{\pi}^{2} M_{\pi}^{2}$ obtained from Eq. (21), and get

$$
\frac{M_{\sigma}^{2}}{M_{\pi}^{2}}=1-\frac{8 N}{m} \frac{\sum_{n=-\infty}^{+\infty}(-)^{n} \int \frac{d^{4} k}{(2 \pi)^{4}} \frac{\left(m+\bar{\Sigma}_{s}\right)^{2} \bar{\Sigma}_{s}^{2}}{\left[\bar{k}^{2}+\left(m+\bar{\Sigma}_{s}\right)^{2}\right]^{2}} e^{i n \frac{k_{0}}{T}}}{\langle\bar{\psi} \psi\rangle_{T, \mu}-4 N \sum_{n=-\infty}^{+\infty}(-)^{n} \int \frac{d^{4} k}{(2 \pi)^{4}}\left[\frac{\bar{\Sigma}_{s}}{\bar{k}^{2}+\left(m+\bar{\Sigma}_{s}\right)^{2}}-\frac{\bar{s}}{s_{0}} \frac{\Sigma_{0}}{\bar{k}^{2}+\Sigma_{0}^{2}}\right] e^{i n \frac{k_{0}}{T}}}
$$

As we have said, it is particularly interesting to evaluate when, on the phase diagram, the mass of the sigma becomes smaller than twice the pion mass, so that it cannot kinematically decay into its main decay channel. The phase diagram is now of a more complicated structure than in absence of chemical potential. As we know, we expect a tricritical point $\left(\mu_{t}, T_{t}\right)$ separating the region of second order transition from that of first order. We thus have to consider different cases. For $\mu>\mu_{t}$, by letting $T$ grow, one passes through the first order branch of the critical line. For $\mu<\mu_{t}$, increasing $T$ one passes through the second order branch.

We recall that of the two parameters present in the coupling, $\zeta$ is left undetermined and can be only constrained within some limited region from qualitative considerations. These are constraints on the critical values of the chemical potential at very low temperatures, which suggest to locate this parameter in the range $\zeta \simeq 0.1-0.3$ to get reasonable values for the critical quark densities [26]. At the same time the location of the tricritical point as well as the appearance of a minimum in the origin (namely the line of second order phase transitions and its continuation at low temperatures) are very few affected by the choice of this parameter. To be specific, the coordinates of the tricritical point $\left(\mu_{t}, T_{t}\right)$ (in $\left.M e V\right)$ change from $(76,92)$ for $\zeta=0.1$ to $(75,91)$ for $\zeta=0.3$. 
To evaluate $M_{\sigma}^{2} / M_{\pi}^{2}$ in Eq. (26) we can perform the integration in $k_{0}$ by using the Cauchy theorem and summing up the series; then, through a numerical calculation we can finally perform the integration left. Typical behaviours of the ratio $M_{\sigma} / M_{\pi}$ at fixed $\mu$ and growing $T$ for $\mu<\mu_{t}$ and $\mu>\mu_{t}$ are shown in Fig. 1 and Fig. 2. We have chosen $\zeta=0.1$, but almost the same pictures show for other values of $\zeta$ in the range $0.1-0.3$. Thus Fig. 1 is for $\mu=70 \mathrm{MeV}\left(<\mu_{t}\right)$, whereas Fig. 2 is for $\mu=120 \mathrm{MeV}\left(>\mu_{t}\right)$. The current quark mass is set to $m=\left(m_{u}+m_{d}\right) / 2=9.5 \mathrm{MeV}$. The little jump visible in Fig. 2, which occurs after the $\sigma$ mass has decreased below twice the pion mass, is due to the jump in the fermion condensate when crossing the line of first order phase transitions. The size of this jump increases for higher values of the chemical potential, namely by going far from the tricritical point.

\section{CONCLUSIONS}

Clear-cut experimental signatures for the sigma meson in low energy QCD phenomenology are made difficult by its expected large width and by complicated unitarity contributions and related distortions. We have examined here the picture emerging at finite temperatures and densities. In the simplest model with massless $u$ and $d$ quarks the pion and the sigma are degenerate in mass only as long as chiral symmetry holds. At zero temperature and in absence of chemical potential, chiral symmetry is broken, the pion is a goldstone of vanishing mass, and the sigma has has a finite mass from the chiral condensate. In the temperature-density phase diagram one expects that, when approaching, from the chirally broken phase, the line of chiral restoration, the pion mass increases and the sigma mass decreases, and finally the two particles become mass degenerate after passing into the region of chiral restoration. Before reaching the transition line, within a certain strip touching such a line, the sigma will not have phase space left to decay into two pions. The dominant decay channel will be that into two photons, and in such ideal conditions the signal from a sigma will be a narrow bump in the two-photon invariant mass. The ideal picture will be quantitatively changed because of the finite $u$ and $d$ quark masses and the role of the strange 
quark, but the qualitative feature of a narrow sigma resonance is expected to remain there even after inclusion of these effects. It is too early to say whether a possible experimental signature for the chiral restoring transition may be inferred from such behaviour. On the other hand determination of the narrow two-photon bump would be of great theoretical interest within the chiral picture.

The present calculation relies on the composite operator formalism applied to QCD at finite temperature and chemical potential. It uses the same QCD model parameters that had been derived in previous works, using the same formalism, for the masses and decay constants of the pseudoscalar mesons. The experimental inputs were the mass and decay constant of the pion, the charged kaon mass and the electromagnetic mass difference between the neutral and the charged kaon. The effective potential is consistently renormalized to derive expressions for the masses of the scalar and pseudoscalar mesons at finite temperature and chemical potential. We have limited the calculation to the phase region where quarkantiquark condensates dominate (no quark-quark condensates). The ratio of the sigma mass to the pion mass is then calculated within the composite operator formalism for finite temperatures and densities. We recall that the same model employed here had produced in the plane of temperature and chemical potential a second order transition for low chemical potentials and one of first order beyond some critical value of the chemical potential (corresponding to a so-called tricritical point). This feature induces a related discontinuity in the ratio of pion to sigma mass when going beyond the first order transition, that is for higher densities. As to the determination of the region in the phase diagram where the sigma is no longer kinematically able to decay into two pions, we have calculated it quantitatively within the model and found that, as expected,it is indeed confined to a small strip near the transition line, and this independently of of the type of transition. We have discussed in detail the dependence on the thermal parameters and drawn the expected phase diagram behaviours. 


\section{REFERENCES}

[1] Y. Nambu, Phys. Rev. Lett. 4, 380 (1960).

[2] T. D. Lee and G. C. Wick, Phys. Rev. 9, 2291 (1974).

[3] L. Dolan and R. Jackiw, Phys. Rev. D9, 3320 (1974); C. W. Bernard, Phys. Rev. D9, $3312(1974)$.

[4] S. Weinberg, Phys. Rev. D9, 3357 (1974).

[5] For general reviews and references see: H. Satz, H. J. Specht and R. Stock, Proceedings of the International Conference "Quark Matter 87", Z. Phys. C38, 1 (1988); M. Jacob,Proceedings of the International Conference "Quark Matter 88", Nucl. Phys. 498A, 1 (1989); P. Carruthers and J. Rafelski, Proceedings of the International Conference "Hadronic Matter in Collision" Tucson Arizona,World Scientific, Singapore (1989); E. V. Shuryak, "The QCD vacuum", World Scientific, Singapore (1988); D. Gross, R. Pisarski, and L. Yaffe, Rev. Mod. Phys. 53, 43 (1981); J. Cleymans, R. V. Gavai, E. Suhonen, Phys. Rep. 130, 217 (1986); L. McLerran, Rev. Mod. Phys. 58, 1021 (1986); F. Karsch, Z. Phys. C38, 147 (1988).

[6] For reviews and references see: A. D. Linde, Rep. Prog. Phys. 42, 389 (1979); B. Sinha and S. Raha, Proceedings of the International Conference on Physics and Astrophysics of Quark Gluon Plasma, World Scientific, Singapore (1988).

[7] M.A. Stephanov in the proceedings of Workshop on QCD at Finite Baryon Density: A Complex System with a Complex Action, Bielefeld, Germany, 27-30 Apr 1998, hepph/9806509; J. Berges. MIT-CTP-2758, Talk given at Workshop on QCD at Finite Baryon Density: A Complex System with a Complex Action,Bielefeld, Germany, 27-30 Apr 1999, hep-ph/9807362; K. Rajagopal, Talk given at 3rd Workshop on Continuous Advances in QCD (QCD 98), Minneapolis, MN, 16-19 Apr, 1998, hep-ph/9808348; M.Harada and A. Shibata, IFP-762-UNC, Jul 1998 hep-ph/9807408. 
[8] K. Rajagopal, The Phases of QCD in Heavy Ion Collisions and Compact Stars, to appear in the Comments of Nuclear and Particle Physics section of Comments on Modern Physics, hep-ph/0009058

[9] F. Karsch, Lattice 93, Nucl. Phys. (Proc. Suppl.) B34, 63 (1994).

[10] V. Bernard and U.G.Meissner, Phys. Rev. D38, 1531 (1988); M.K.Volkov, Phys. Part. Nucl.,24, 35 (1993); V. Vogel and W. Weise, Progr. Part. and Nucl. Phys. 27, 195, 1991; J.Hufner, S.P. Klevansky, P. Zhuang, and H.Voss, Ann. Phys. (N.Y.) 234, 225 (1994); T. Hatsuda and T. Kunihiro, Phys. Lett. 185B, 305 (1987); Prog. Theo. Phys. Suppl. 91, 284 (1987); Phys. Rep. 247, 221 (1994); W. Florkowski, Acta Phys. Polon. B28, 2079 (1997).

[11] A. Barducci, R. Casalbuoni, S. De Curtis, R. Gatto and G. Pettini, Phys. Lett. B244, $311(1990)$.

[12] A. Barducci, R. Casalbuoni, S. De Curtis, D. Dominici and R. Gatto, Phys. Rev. D38, 238 (1988).

[13] A. Barducci, R. Casalbuoni, S. De Curtis, R. Gatto and G. Pettini, Phys. Rev. D46, 2203 (1992).

[14] R. D. Pisarski, "Workshop on Finite Temperature QCD and Quark-Gluon Transport Theory", Wuhan, China, 1994; e-Print Archive: hep-ph/9503330.

[15] J. Weinstein and N. Isgur, Phys. Rev.D27 (1983) 588; K. L. Au, D. Morgan and M. R. Pennington, Phys. Rev.D35 (1987) 1633; J. Weinstein and N. Isgur, Phys. Rev.D41 (1990) 2236; J. Gasser and U.-G. Meißner, Nucl. Phys.B357 (1991) 90; U. G. Meißner, Comm. Nucl. Part. Phys.20 (1991) 119; G. Janssen, B.C. Pearce, K. Holinde and J. Speth, Phys. Rev.D52 (1995); N. A. Tornqvist and M. Roos, Phys. Rev. Lett.76(1996) 1575; J. A. Oller and E. Oset, Phys. Rev. D60 (1999) 074023.

[16] E.M.Aitala et al. hep-ex/0007028. 
[17] R. Gatto, G. Nardulli, A.D. Polosa, and N.A. Tornqvist, hep-ph/0007207.

[18] N. A. Tornqvist, Summary talk at the conference on the sigma resonance, conference: "Possible Existence of the Light Sigma Resonance and its Implications to Hadron Physics", Yukawa Institute for Theoretical Physics, Kyoto, Japan, June 2000, hep$\mathrm{ph} / 0008135$

[19] M. R. Pennington, Talk given at the 13th International Workshop on Photon-Photon Collisions (Photon2000), September 2000, Ambleside, U. K. hep-ph/0009267

[20] A. Barducci, R. Casalbuoni, S. De Curtis, R. Gatto and G. Pettini, Phys. Lett. B231, 463 (1989); Phys. Rev. D41, 1610 (1990).

[21] R. D. Pisarski presented at the 'Workshop on Finite Temperature QCD and QuarkGluon Transport Theory', Wuhan, China, Apr 1994, hep-ph/9503330; Y. Taniguchi, Y. Yoshida, Phys.Rev.D55:2283,1997; O. Kiriyama, M. Maruyama, F. Takagi, Phys.Rev.D58:116001,1998 and hep-ph/0001108; M. Stephanov, K. Rajagopal, E. Shuryak, Phys.Rev.D60:114028,1999; K. Rajagopal,in the proceedings of Conference on Strong and Electroweak Matter (SEWM 98), Copenhagen, Denmark, Dec 1998, hepph/9903547 and proceedings of International Conference on Quark Nuclear Physics (QNP2000), Adelaide, Australia, February 2000, hep-ph/0005101; M. Harada , A Shibata, Phys.Rev.D59:014010,1999; J.J.M. Verbaarschot, T. Wettig to appear in Ann.Rev.Nucl.Part.Sci, hep-ph/0003017.

[22] A. Barducci, R. Casalbuoni, R. Gatto, M.Modugno and G. Pettini, Phys. Rev. D59, 114024 (1999).

[23] R. Casalbuoni, in Proceedings of the International Symposium on Composite Models of Quarks and Leptons, Tokyo, Japan, 1985, edited by H. Terazawa and M. Yasué (Institute for Nuclear Study, University of Tokyo, Tokyo, 1985).

[24] A. Barducci, R. Casalbuoni, S. De Curtis, R. Gatto and G. Pettini, Phys. Lett. B240, 
429 (1990).

[25] J. Gasser and H. Leutwyler, Phys. Lett. B184, 83 (1987); B188, 477 (1987).

[26] A. Barducci, R. Casalbuoni, R. Gatto and G. Pettini, Phys. Rev. D49, 426 (1994). 


\section{FIGURES}

FIG. 1. Plot of $M_{\sigma} / M_{\pi}$ versus $T$ for $m=9.5 \mathrm{MeV}$. The chemical potential is fixed at $\mu=70 \mathrm{MeV}$, which is below the value $\mu=\mu_{t}=76 \mathrm{MeV}$ at the tricritical point, for $\zeta=0.1$.

FIG. 2. Plot of $M_{\sigma} / M_{\pi}$ versus $T$ for $m=9.5 \mathrm{MeV}$. The chemical potential is fixed at $\mu=120 \mathrm{MeV}$, which is above the value $\mu=\mu_{t}=76 \mathrm{MeV}$ at the tricritical point, for $\zeta=0.1$. 


\section{$\mathrm{M}_{\sigma} / \mathrm{M}_{\pi}$}

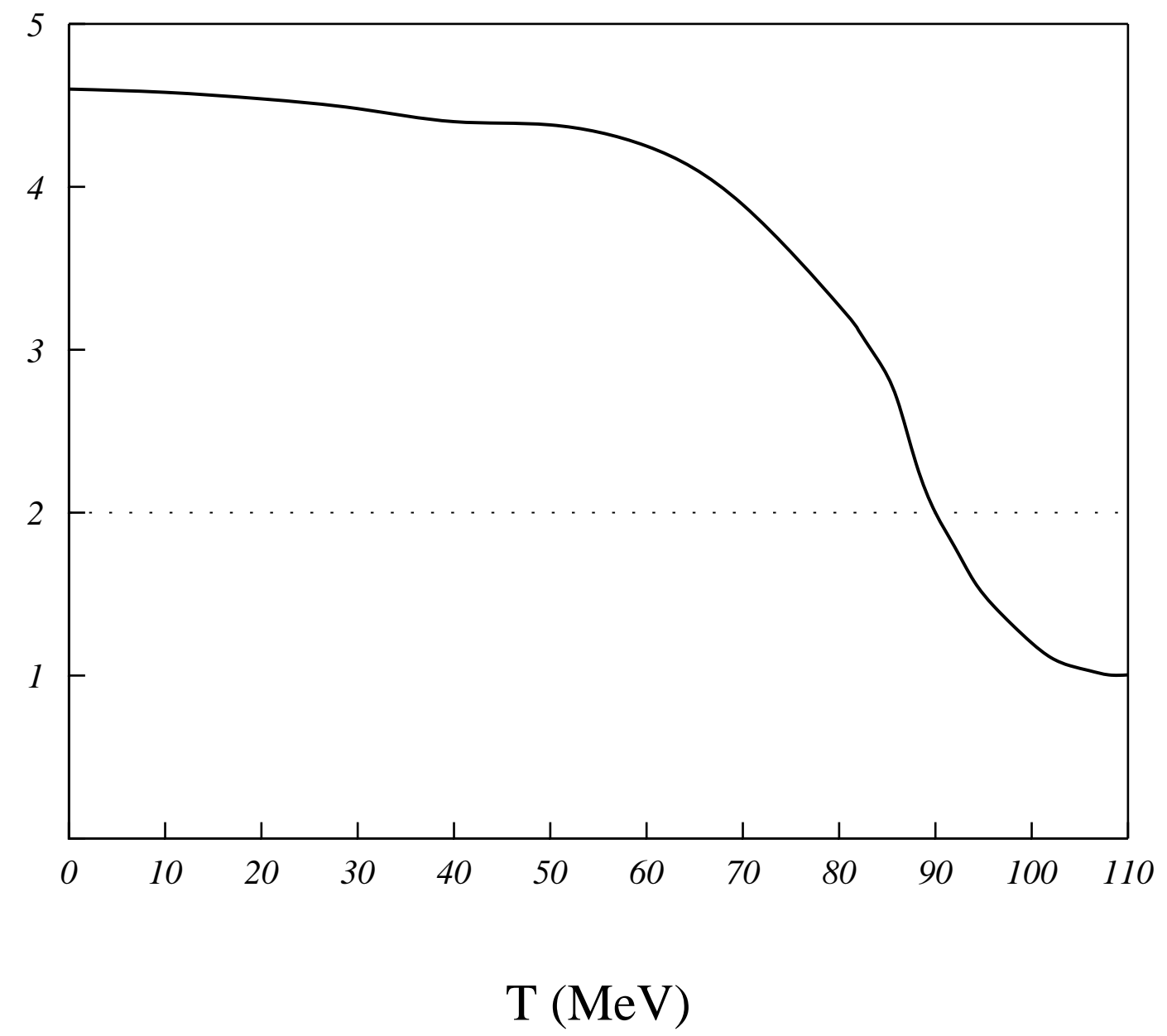

Fig. 1 


\section{$\mathrm{M}_{\sigma} / \mathrm{M}_{\pi}$}

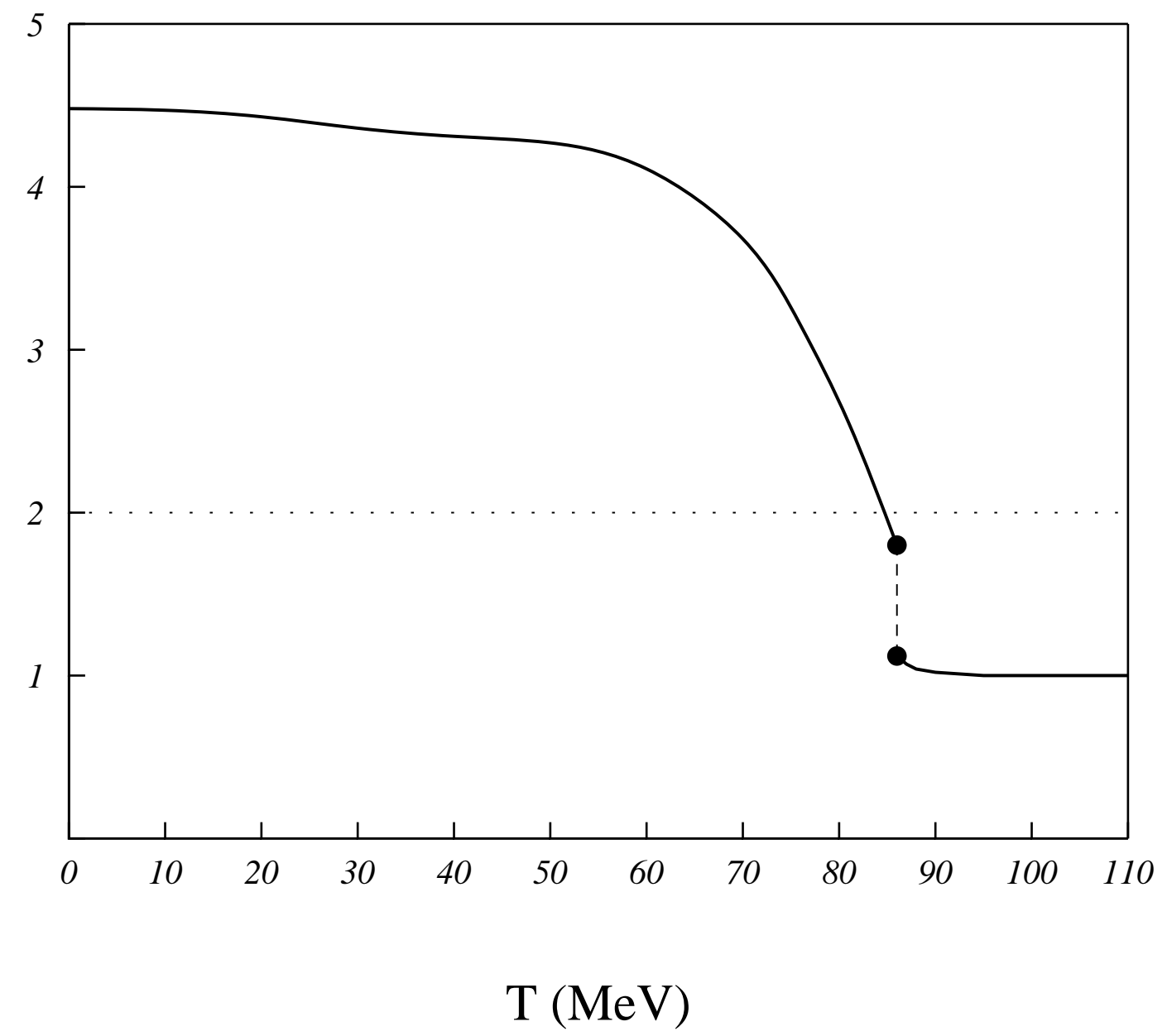

Fig. 2 